Pacific Journal of Mathematics

ON GENERALIZED CAYLEY-DICKSON ALGEBRAS 


\title{
ON GENERALIZED CAYLEY-DICKSON ALGEBRAS
}

\author{
ROBERT B. BROWN
}

\begin{abstract}
Among those algebras whose multiplication does not satisfy the associative law is a particular family of noncommutative Jordan algebras, the generalized Cayley-Dickson algebras. These are certain central simple algebras whose dimensions are all powers of two. Most of this paper is concerned with giving the classification up to isomorphism of those of dimensions 16, 32, and 64 and determining the automorphism groups. In addition to this some generalized Cayley-Dickson division algebras are constructed. Precise criteria for when the 16dimensional algebras are division algebras are formulated and applied to algebras over some common fields. For higher dimensions no such criteria are given. However, specific examples of division algebras for each dimension $2^{t}$ are constructed over power-series fields.
\end{abstract}

Definitions. Let us recall the definition of our algebras. Let $\mathfrak{A}$ be any algebra (not necessarily associative) with an involution $a \rightarrow a^{*}$, that is, a nonsingular linear transformation on $\mathfrak{A}$ such that $(a b)^{*}=b^{*} a^{*}$ and $\left(a^{*}\right)^{*}=a$. If $\gamma$ is a nonzero element of the ground field, we define the algebra $\mathfrak{A}\{\gamma\}$ to be the set of pairs $(a, b)$ with $a$ and $b$ in $\mathfrak{A}$ and with addition and scalar multiplication defined in the obvious way. To avoid confusion with bilinear forms which will be appearing let us write $u$ for $(0,1)$ and $a+b u$ for $(a, b)$. Multiplication in $\mathfrak{A}(\gamma)$ is then defined by

$$
(a+b u)(c+d u)=\left(a c+\gamma d^{*} b\right)+\left(d a+b c^{*}\right) u .
$$

The map $a \rightarrow a+0 u$ imbeds $\mathfrak{A}$ isomorphically in $\mathfrak{A}\{\gamma\}$, and $a+b u \rightarrow$ $a^{*}-b u$ extends the involution to $\mathfrak{A}\{\gamma\}$. If 1 is a unity element of $\mathfrak{A}$, then $1+0 u$ is a unity of $\mathfrak{A}\{\gamma\}$.

A generalized Cayley-Dickson algebra $\mathfrak{A}_{t}$ of dimension $2^{t}$ is constructed by choosing nonzero elements $\gamma_{1}, \cdots, \gamma_{t}$ in the ground field $\mathfrak{F}$. Then we set $\mathfrak{A}_{0}=\mathfrak{F}$ (with the trivial involution $a^{*}=a$ ) and $\mathfrak{A}_{i}=\mathfrak{A}_{i-1}\left\{\gamma_{i}\right\}=\mathfrak{A}_{i-1} \oplus \mathfrak{A}_{i-1} u_{i}$ for $i=1,2, \cdots, t$. The norm $n(x)=$ $x x^{*}=x^{*} x$ of $x$ in $\mathfrak{A}_{t}$ is a multiple of the unity element and can be linearized to give a nondegenerate bilinear form on $\mathfrak{A}_{t}$ :

$$
\begin{aligned}
(x, y) & =\frac{1}{2}[n(x+y)-n(x)-n(y)] \\
& =\frac{1}{2}\left(x y^{*}+y x^{*}\right) .
\end{aligned}
$$

We note that if $n(x) \neq 0$, then $x$ has the inverse $n(x)^{-1} x^{*}$ and 
$n\left(x^{-1}\right)=n(x)^{-1}$. For $\mathfrak{N}_{3}$ the norm can be seen easily to be equivalent to

$$
\left(x_{1}^{2}-\gamma_{1} x_{2}^{2}\right)-\gamma_{2}\left(x_{3}^{2}-\gamma_{1} x_{4}^{2}\right)-\gamma_{3}\left[\left(x_{5}^{2}-\gamma_{1} x_{6}^{2}\right)-\gamma_{2}\left(x_{7}^{2}-\gamma_{1} x_{8}^{2}\right)\right],
$$

where the first term comes from the restriction of the norm to $\widetilde{\gamma} 1$ and the other seven terms from the restriction to the subspace $\mathfrak{V}_{3}^{0}$ of all $x$ such that $(x, 1)=0$. Also for $t \leqq 3$ only, $\mathfrak{A}_{t}$ is a composition algebra: $n(x y)=n(x) n(y)$. (See [2].) The algebras $\mathfrak{A}_{2}$ are generalized quaternions and $\mathfrak{U}_{3}$ the usual Cayley-Dickson algebras.

Suppose that $\mathfrak{A}_{0}, \mathfrak{A}_{1}, \cdots, \mathfrak{A}_{t-1}$ is a sequence of subalgebras of $\mathfrak{A}_{t}$ and $v_{1}, \cdots, v_{t}$ is a set of generators for $\mathfrak{A}_{t}$ such that $v_{i}^{2}=\delta_{i} \neq 0$, $\mathfrak{F}=\mathfrak{A}_{0} \subset \cdots \subset \mathfrak{A}_{t-1} \subset \mathfrak{A}_{t}$, dimension $\mathfrak{U}_{i}=2^{i}$, and $\mathfrak{A}_{i}=\mathfrak{A}_{i-1}+\mathfrak{N}_{i-1} v_{i}$. Suppose further that multiplication in $\mathfrak{A}_{i}$ can be written as

$$
\left(a+b v_{i}\right)\left(c+d v_{i}\right)=\left(a c+\delta_{i} d^{*} b\right)+\left(d a+b c^{*}\right) v_{i} .
$$

Then we will call $v_{1}, \cdots, v_{t}$ a normal set of generators for $\mathfrak{A}_{t}$. In general, $\mathfrak{A}_{t}$ has normal sets of generators other than the set $u_{1}, \cdots, u_{t}$ used in the original construction of $\mathfrak{U}_{t}$. We recall that for $\mathfrak{U}_{3}$ all normal sets of generators can be found in the following way [2, pages 6-8]. We choose $v_{1}$ to be any element of $\mathfrak{A}_{3}$ such that $\left(v_{1}, 1\right)=0$ and $v_{1}^{2} \neq 0$. For $v_{2}$ we then choose any element such that $\left(v_{2}, 1\right)=\left(v_{2}, v_{1}\right)=0$ and $v_{2}^{2} \neq 0$. Finally, $v_{3}$ can be any element such that $\left(v_{3}, 1\right)=\left(v_{3}, v_{1}\right)=$ $\left(v_{3}, v_{2}\right)=\left(v_{3}, v_{1} v_{2}\right)=0$ and $v_{3}^{2} \neq 0$. The known property [2] that $\mathfrak{H}_{3}$ has zero divisors if and only if it has an element $a \neq 0$ such that $n(a)=a a^{*}=0$ will be used in $\S 3$. The multiplication table for $\mathfrak{A}_{t}$ in terms of a normal set of generators can be determined from equation (2). In particular, for $\mathfrak{H}_{3}$ we have the following products, which we will use in $\S 3: \quad\left(v_{1} v_{2}\right)\left(v_{1} v_{3}\right)=\left(v_{1}^{2}\right)\left(v_{2} v_{3}\right), v_{3}\left(v_{1} v_{2}\right)=-\left(v_{1} v_{2}\right) v_{3}, v_{2}\left(v_{1} v_{3}\right)=$ $\left(v_{1} v_{2}\right) v_{3}, v_{3} v_{2}=-v_{2} v_{3}$, and $v_{1}\left(v_{2} v_{3}\right)=-\left(v_{1} v_{2}\right) v_{3}$.

In $\mathfrak{A}_{t}$ let $S$ be a subset of $T=\{4, \cdots, t\}$ and let $a$ be in the 8-dimensional subalgebra generated by $u_{1}, u_{2}$, and $u_{3}$. If the integers $i, j, \cdots$ of $S$ are written in increasing order, we define $a_{S}$ to be $\left(\cdots\left(\left(a u_{i}\right) u_{j}\right) \cdots\right)$. Then every element $x$ in $\mathfrak{A}_{t}$ can be written uniquely in the form $\sum_{S \subseteq T} a_{S}$.

2. Automorphisms. The structure of the automorphism groups of the algebras $\mathfrak{A}_{3}$ can be found in [2]. To compute Aut $\left(\mathfrak{A}_{t}\right)$ for $t \geqq 4$ we will need Schafer's result [4] that for $t \geqq 4$ the derivations $\mathfrak{D}\left(\mathfrak{\Re}_{t}\right)$ of $\mathfrak{U}_{t}$ are all of the form $a+b u \rightarrow a D+(b D) u$, where $D$ is a derivation of $\mathfrak{U}_{t-1}$. If $\mathfrak{S}^{*}$ denotes the enveloping algebra (not necessarily with unity element) of a set $\mathfrak{S}$ of linear transformations on a vector space, then for $t \geqq 4$ any element of $\mathfrak{D}\left(\mathfrak{A}_{t}\right)^{*}$ is of the form $a+b u \rightarrow$ $a E+(b E) u$, where $E$ is in $\mathfrak{D}\left(\mathfrak{A}_{t-1}\right)^{*}$. 
Let $\mathfrak{A}_{t}=\mathfrak{A}_{t-1}\{\gamma\}$ and assume that the ground field contains $\beta=\sqrt{(-3 \gamma)}$. Let $\theta$ be an automorphism of $\mathfrak{A}_{t \rightarrow-1}$. Then we can readily check that the following transformations are automorphisms of $\mathfrak{A}_{t}$.

$$
\begin{array}{rlrl}
\theta^{\prime}: & a+b u & \rightarrow a \theta+(b \theta) u, & \\
\varepsilon: & a+b u & \rightarrow a-b u . \\
\psi: & a+b u & \rightarrow \frac{1}{4}\left[a+3 a^{*}+\beta\left(b-b^{*}\right)\right] \\
& & +\frac{1}{4}\left[b+3 b^{*}+\beta \gamma^{-1}\left(a-a^{*}\right)\right] u .
\end{array}
$$

The following products are also easily checked: $\psi \theta^{\prime}=\theta^{\prime} \psi, \varepsilon \theta^{\prime}=\theta^{\prime} \varepsilon$, $\varepsilon^{2}=1, \psi^{3}=1, \varepsilon \psi=\psi^{2} \varepsilon$ (composition reads from left to right). Therefore Aut $\left(\mathfrak{A}_{t}\right)$ has a subgroup $G$ isomorphic to Aut $\left(\mathfrak{U}_{t-1}\right) \times S_{3}$, where $S_{3}$ is the symmetric group of degree three. If the ground field does not contain $\beta$, then $\psi$ does not exist and Aut $\left(\mathfrak{H}_{t}\right)$ has a subgroup $G$ isomorphic to Aut $\left(\mathfrak{A}_{t-1}\right) \times S_{2}$, where $S_{2}$ is the group of order two. Here are the two main theorems.

TheOREm 1. For $t=4,5,6$ and for ground fields of characteristics not two or three, Aut $\left(\mathfrak{A}_{t}\right)=G$.

THEOREM 2. For $t=4,5,6$ and for ground fields of characteristics not two or three $\mathfrak{N}_{t}=\mathfrak{N}_{t-1}\{\gamma\}$ and $\mathfrak{H}_{t}^{\prime}=\mathfrak{U}_{t-1}^{\prime}\left\{\gamma^{\prime}\right\}$ are isomorphic if and only if $\mathfrak{P}_{t-1}$ and $\mathfrak{X}_{t-1}^{\prime}$ are isomorphic and $\gamma=\lambda^{2} \gamma^{\prime}$ for $\lambda$ in the ground field.

We will prove these theorems in detail here for $t=4$ only and will sketch the proof for $t=5,6$. We conjecture that both theorems are true for all $t \geqq 4$.

First we provide an elementary characterization of $u=u_{t}$.

Lemma 1. If $v$ is an element of $\mathfrak{U}_{t}(t \geqq 4)$ sush that $(v, 1)=0$ and

$$
x(x v)=x^{2} v, \quad(v x) x=v x^{2}
$$

for all $x$ in $\mathfrak{U}_{t}$, then $v$ is a multiple of $u$.

Proof. Let $x=a+b u$ and $v=c+d u$. After expanding (3), among the conditions we obtain on $c$ and $d$ by equating appropriate terms are $a^{*}\left(d^{*} b\right)=\left(a^{*} d^{*}\right) b$ and $b\left(c^{*} a^{*}\right)=\left(b c^{*}\right) a^{*}$. Hence, $c$ and $d$ are in the middle nucleus of $\mathfrak{A}_{t-1}$, which contains only scalar multiples of 1 [4, Equation 14b]. Then since $(v, 1)=0, c=0$. This proves Lemma 1.

Our knowledge of $\mathfrak{D}\left(\mathfrak{U}_{t}\right)$ can be used to advantage with the follow- 
ing lemma about $\mathfrak{D}\left(\mathfrak{U}_{3}\right)$.

LEMMA 2. $\mathfrak{D}\left(\mathfrak{\Re}_{3}\right)$ maps $\mathfrak{A}_{3}$ into $\mathfrak{H}_{3}^{0}$. Furthermore, $\mathfrak{D}\left(\mathfrak{H}_{3}\right)^{*}$ acts as the full set of linear transformations on $\mathfrak{U}_{3}^{0}$.

Proof. Let $w_{1}, \cdots, w_{7}$ be a basis of $\mathfrak{U}_{3}^{0}$ such that $w_{i}^{2} \neq 0$ and $\left(w_{i}, w_{j}\right)=0$ if $i \neq j$. Then if $i \neq j$ there is some $k$ such that the ordered triple $L=\left\{v_{1}=w_{i}, v_{2}=w_{j}, v_{3}=w_{k}\right\}$ is a normal set of generators for $\mathfrak{A}_{3}$. Then a short check will show that the map $D_{L}$ such that $1 D_{L}=0, v_{1} D_{L}=v_{2}, v_{2} D_{L}=-n\left(v_{2} v_{1}^{-1}\right) v_{1},\left(v_{1} v_{2}\right) D_{L}=0$ is a derivation of $\mathfrak{A}_{2}$. This can be extended to $\mathfrak{A}_{3}$ by setting $v_{3} D_{L}=0$. Then

$$
\left(v_{1} v_{3}\right) D_{L}=v_{2} v_{3},\left(v_{2} v_{3}\right) D_{L}=-n\left(v_{2} v_{1}^{-1}\right) v_{1} v_{3},
$$

and $\left(v_{1} v_{2}\right) v_{3} D_{L}=0$. The triples $M=\left\{v_{2}, v_{3}, v_{1}\right\}$ and $N=\left\{v_{3}, v_{2}, v_{1} v_{3}\right\}$ are also normal sets of generators. If $E_{i j}=D_{L} D_{M} D_{N}$ in $\mathfrak{D}\left(\mathfrak{H}_{3}\right)^{*}$, then $w_{n} E_{i n}=\delta_{i j} w_{j}$. These $E_{i j}$ generate the full set of linear transformations on $\mathfrak{2}_{3}^{0}$. To finish the proof of Lemma 2 it is sufficient to show that if $D$ is in $\mathfrak{D}\left(\mathfrak{A}_{3}\right),(a, 1)=0$. and $a^{2}=\beta 1 \neq 0$, then $(\alpha D, 1)=0$. Let $a D=\alpha 1+b$, where $(b, 1)=0$. Then

$$
0=a^{2} D=(a D) a+a(a D)=2 \alpha a+(b a+a b)=2 \alpha a-(a, b) 1 .
$$

Therefore $\alpha=0$. This proves Lemma 2 .

We turn first to the proof of Theorem 2. Let $\varphi$ be an isomorphism of $\mathfrak{A}_{t}$ onto $\mathfrak{X}_{t}^{\prime}$. Lemma 1 assures us that $u_{t} \varphi=\lambda u_{t}^{\prime}$. Hence $\left(u_{t}^{2}\right) \varphi=$ $\left(\gamma_{t} 1\right) \varphi=\gamma_{t} 1^{\prime}=\lambda^{2}\left(u_{t}^{\prime}\right)^{2}=\lambda^{2} \gamma_{t}^{\prime} 1^{\prime}$ and $\gamma_{t}=\lambda^{2} \gamma_{t}^{\prime}$. For $a \neq 0$ in $\mathfrak{A}_{3}$ such that $(a, 1)=0$, let

$$
a \varphi=\sum_{S \equiv T}\left(a \eta_{S}\right)_{S}
$$

where the $\eta_{S}$ are linear transformations of $\mathfrak{A}_{3}$ into $\mathfrak{2}_{3}^{\prime}$. Evidently $\varphi^{-1} \mathfrak{D}\left(\mathfrak{U}_{t}\right)^{*} \varphi=\mathfrak{D}\left(\mathfrak{U}_{t}^{\prime}\right)^{*}$. Therefore $\mathfrak{U}_{3}^{\prime}$ is spanned by 1 and

$$
\sum\left(a \mathfrak{D}\left(\mathfrak{U}_{3}\right) * \eta_{S}\right)_{S}=a \mathfrak{D}\left(\mathfrak{H}_{t}\right)^{*} \varphi=a \varphi \mathfrak{D}\left(\mathfrak{A}_{t}^{\prime}\right)^{*}=\sum\left(a \eta_{S} \mathfrak{D}\left(\mathfrak{U}_{3}^{\prime}\right)^{*}\right)_{S} .
$$

The preceding equation and Lemma 2 imply that $\left(a \eta_{s}, 1^{\prime}\right)=0$ and if $a \eta_{s}=0$ for some $a$, then $\eta_{s}=0$.

We fix some $R \cong T$ such that $\eta_{R} \neq 0$ and denote $\eta_{R}$ by $\eta$. Now we will show that for all $U \leqq T, \eta_{U}=\alpha_{U} \eta$, for $\alpha_{U}$ in the ground field. If for some $a, a \eta_{U}$ is not a multiple of $a \eta$, we find $E^{\prime}$ in $\mathfrak{D}\left(\mathfrak{H}_{3}^{\prime}\right)^{*}$ such that $a \eta_{V} E^{\prime}=0$ and $a \eta E^{\prime} \neq 0$. Since there exists an $E$ in $\mathfrak{D}\left(\mathfrak{A}_{3}\right)^{*}$ such that $\sum\left(a E \eta_{S}\right)_{S}=\sum\left(a \eta_{S} E^{\prime}\right)_{S}, a \eta E^{\prime} \neq 0$ implies that $a E \neq 0$, and $a E \eta_{U}=0$ implies that $\eta_{U}=0$. Hence $\alpha_{U}=0$ will do for $\alpha_{U}$. Now we can write $a \varphi=\sum\left(\alpha_{S} a \eta\right)_{S}$. To show that $\alpha_{S}$ does not depend on $a$, let $b_{\varphi}=\sum\left(\beta_{S} b \eta\right)_{s}$. Choose $E$ in $\mathfrak{D}\left(\mathfrak{H}_{3}\right)^{*}$ such that $a E=b$. Then 


$$
\sum\left(\beta_{S} b \eta\right)_{S}=b \varphi=a \varphi\left(\varphi^{-1} E \varphi\right)=\sum\left(\alpha_{S} a \eta E^{\prime}\right)_{S}
$$

for some $E^{\prime}$ in $\mathfrak{D}\left(\mathfrak{A}_{3}^{\prime}\right)^{*}$. Since $\alpha_{R}=\beta_{R}=1, b \eta=a \eta E^{\prime}$. Therefore $\alpha_{S}=\beta_{S}$ for all $S$. We know that $(a \eta, 1)=0$. Also, $(a \eta, b \eta)=0$ if and only if $(a, b)=0$. To see this choose $a$ such that $n(a) \neq 0$. Then $n(\alpha)=n(a \varphi)=\alpha n(a \eta) \neq 0$, for some nonzero constant $\alpha$ which does not depend on $a$. Linearizing, we obtain $(a, b)=\alpha(a \eta, b \eta)$. Finally for $i \geqq 4, u_{i} \varphi=\sum a_{S}^{\prime}$ for $a^{\prime}$ in $\mathfrak{X}_{3}^{\prime}$. Since $u_{i} \mathfrak{D}\left(\mathfrak{R}_{t}\right)^{*}=0, a^{\prime} \mathfrak{D}\left(\mathfrak{U}_{3}^{\prime}\right)^{*}=0$ and every $a^{\prime}$ is a scalar multiple of $1^{\prime}$. Altogether,

$$
\begin{aligned}
& a \varphi=\sum \alpha_{S}(a \eta)_{S}, \\
& u_{i} \varphi=\sum \beta_{i S} 1_{S}^{\prime} .
\end{aligned}
$$

Substitution of (4) and (5) into $(x y) \varphi=(x \varphi)(y \varphi)$ for all $x, y$ in $\mathfrak{A}_{t}$ yields a set of quadratic equations that can be used to verify Theorems 1 and 2. Their solution for $t=4$, which we now give in detail, is the easiest. Let $u_{4}=u$, and for $a$ in $\mathfrak{A}_{3}$ let $a \varphi=\alpha(a \eta)+\beta(a \eta) u^{\prime}$, where $\alpha=0$ or 1 . If $\alpha=0, \mathfrak{A}_{3} \varphi$ is spanned by $\mathfrak{A}_{3}^{\prime} u^{\prime}$ and $1^{\prime}$. Since this set is not closed under multiplication, $\alpha=1$. Let $(a, 1)=(b, 1)=$ $(a, b)=0$. Then $(a \eta)^{*}=-a \eta,(b \eta)^{*}=-b \eta$, and $(a \eta)(b \eta)+(b \eta)(a \eta)=$ $-\left[(a \eta)(b \eta)^{*}+(b \eta)(a \eta)^{*}\right]=-(a \eta, b \eta)=0$. Therefore

$$
\begin{aligned}
(a b) \varphi & =(a b) \eta+\beta(a b) \eta u^{\prime} \\
& =\left(a \eta+\beta(a \eta) u^{\prime}\right)\left(b \eta+\beta(b \eta) u^{\prime}\right) \\
& =(a \eta)(b \eta)+\gamma^{\prime} \beta^{2}(b \eta) *(a \eta)+\beta\left[(b \eta)(a \eta)+(a \eta)(b \eta)^{*}\right] u^{\prime} \\
& =(a \eta)(b \eta)+\gamma^{\prime} \beta^{2}(a \eta)(b \eta)-2 \beta((a \eta)(b \eta)) u^{\prime}
\end{aligned}
$$

Hence

$$
\begin{aligned}
& (a b) \eta=\left(1+\gamma^{\prime} \beta^{2}\right)(a \eta)(b \eta), \\
& (a b) \eta=-2(a \eta)(b \eta) .
\end{aligned}
$$

Since these two equations are linear in $a$ and $b$ and true if $(a, b)=0$, they must be true for all $a, b$ in $\mathfrak{2}_{3}^{0}$. Equation (7) implies that $-2 \eta$ is an isomorphism of $\mathfrak{A}_{3}$ onto $\mathfrak{H}_{3}^{\prime}$. This proves Theorem 2 for $t=4$.

To prove Theorem 1 for $t=4$, we assume that $\mathfrak{A}_{4}=\mathfrak{A U}_{4}^{\prime}$. The foregoing arguments show first that $u \varphi= \pm u$. Hence, by replacing $\varphi$ by $\varphi \varepsilon$ if necessary, we may assume that $u \varphi=u$. As before, if $(a, 1)=0$ and $a \neq 0$, then $a \eta \neq 0$. We may also assume that $\beta \neq 0$, for otherwise $\eta$ is already an automorphism of $\mathfrak{A}_{3}$ and $\varphi=\eta^{\prime}$. The remaining computations again lead to equations (6) and (7). Equation (7) shows that $-2 \eta$ is an automorphism of $\mathfrak{A}_{3}$, and equation (6) shows that $\beta=\sqrt{\left(-3 \gamma^{-1}\right)}$. Thus $\varphi=(-2 \eta)^{\prime} \psi \varepsilon$. This proves Theorem 1 for $t=4$. 
3. Division algebras. Over the real field, the rational field, $p$-adic fields, or finite fields, $\mathfrak{A}_{t}$ is never a division algebra. However, we can exhibit division algebras over some other fields. Since $\mathfrak{A}_{t}$ is finite-dimensional, it will be enough to find algebras without zero divisors. The next theorem is about algebras $\mathfrak{A}_{4}$.

THEOREM 3. $\mathfrak{A}_{4}=\mathfrak{2}_{3}\{\gamma\}$ is a division algebra if and only if $\mathfrak{A}_{3}$ is a division algebra, $\gamma$ is not the norm of an element $a$ in $\mathfrak{A}_{3}$ and $-\gamma$ is not the norm of an element $x$ in $\mathfrak{H}_{3}^{0}$.

Proof. The necessity of $\mathfrak{A}_{3}$ being a division algebra is clear. If $\gamma=a a^{*}$, then $(a+u)\left(a^{*}-u\right)=0$. If $-\gamma=x x^{*}$, we extend $x$ to a normal set of generators $v_{1}=x, v_{2}, v_{3}$ of $\mathfrak{A}_{3}$. Then $\left(v_{1} v_{2}-v_{2} u\right)\left(v_{1} v_{3}+\right.$ $\left.v_{3} u\right)=0$. Conversely, suppose that $\mathfrak{A}_{3}$ is a division algebra and $\mathfrak{T}_{4}$ has zero divisors: $(a+b u)(c+d u)=0$. Then $a c+\gamma d^{*} b=0$ and $d a+b c^{*}=0$, and none of $a, b, c, d$ can be zero. Then $c=-\gamma n(a)^{-1} a^{*}\left(d^{*} b\right)$ and $d a=b\left[\gamma n(a)^{-1}\left(b^{*} d\right) a\right]$, so that

$$
b^{*}(d a)=\gamma n\left(b a^{-1}\right)\left(b^{*} d\right) a .
$$

The subalgebra generated by $b, d$, and $a$ is either associative, in which case $\gamma$ is a norm, or is the whole of $\mathfrak{A}_{3}$. In the second case we can obtain a normal set of generators $x, y, z$ by setting

$$
b^{*}=\xi 1+x, d=\eta 1+\alpha x+y, a=\lambda 1+\beta x+\delta y+\varepsilon x y+z,
$$

where

$$
(x, 1)=(y, 1)=(z, 1)=(y, x)=(z, x)=(z, y)=(z, x y)=0 .
$$

Multiplying out (8), we find that in actuality $b^{*}=x, d=y, a=z$. But $x(y z)=-(x y) z$, so that $-\gamma=n\left(a b^{-1}\right)$. Unfortunately these calculations do not generalize to all $\mathfrak{A}_{t}$ because the alternative law (in $\mathfrak{A}_{3}$ ) is used in obtaining (8).

Over a finite field a quaternion algebra $\mathfrak{A}_{2}$ connot be a division algebra, for it would then have to be commutative. Since every $\mathfrak{A}_{t}$ $(t \geqq 2)$ contains an $\mathfrak{U}_{2}$, there are no division algebras $\mathfrak{U}_{t}$ for $t \geqq 2$ over a finite field. Over $p$-adic fields every quadratic form in at least five variables has a nontrivial zero [3, §63]. Therefore, there are no division algebras $\mathfrak{U}_{3}$ or $\mathfrak{P}_{t}(t \geqq 4)$ over a $p$-adic field.

Over the real field or a real algebraic number field let $\mathfrak{P}_{3}$ be the algebra defined by $\gamma_{1}=\gamma_{2}=\gamma_{3}=-1$. By (1) its norm form is equivalent to

$$
n(x)=x_{1}^{2}+x_{2}^{2}+x_{3}^{2}+x_{4}^{2}+x_{5}^{2}+x_{6}^{2}+x_{7}^{2}+x_{8}^{2},
$$

and it is a division algebra because (9) never gives a nontrivial repre- 
sentation of zero over a subfield of the reals. (For the real or rational field this is the only division algebra $\mathfrak{A}_{3}[1, \S 10]$. Next, $\gamma=n(a)$ or $-\gamma=n(x)$ for some $x$ such that $(x, 1)=0$ if and only if the quadratic form (10) or (11) below, respectively, has a nontrivial zero.

$$
\begin{gathered}
n(x)-\gamma x_{9}^{2}=x_{1}^{2}+x_{2}^{2}+x_{3}^{2}+x_{4}^{2}+x_{5}^{2}+x_{6}^{2}+x_{7}^{2}+x_{8}^{2}-\gamma x_{9}^{2} . \\
x_{2}^{2}+x_{3}^{2}+x_{4}^{2}+x_{5}^{2}+x_{6}^{2}+x_{7}^{2}+x_{8}^{2}+\gamma x_{9}^{2} .
\end{gathered}
$$

Over the real field (10) is indefinite and has a zero if $\gamma>0$, (11) if $\gamma<0$. A result of Hasse in algebraic number theory tells us that over an algebraic number field a quadratic form in at least five variables has a nontrivial zero if and only if all of its real conjugate forms are indefinite $[3, \S 66]$. Over the rationals the only real conjugate forms for (10) and (11) are (10) and (11) themselves, one of which is indefinite. Hence, over the real and rational fields there are no division algebras for $\mathfrak{U}_{4}$, thus none for $\mathfrak{U}_{t}(t \geqq 4)$. However, suppose we choose a positive real number $\lambda$ whose square roots are not rational, let $\gamma=\sqrt{\lambda}$ or $-\sqrt{\lambda}$, and let the ground field be the real quadratic extension $\mathfrak{Q}[\gamma]$ of the rational field $\mathfrak{\Omega}$. Then (10) has the real conjugate forms $n(x) \pm \gamma x_{9}^{2}$, one of which is definite. Hence (10) has no nontrivial zeros; similarly, neither does (11). Therefore $\mathfrak{2}_{4}$ is a division algebra.

We can use a different method to construct for every $t$ a division algebra $\mathfrak{A}_{t}$ over a suitable field. Let $\mathfrak{F}$ be any field of characteristic not two, and let $X_{1}, \cdots, X_{t}$ be $t$ algebraically independent indeterminates over $\widetilde{F}$. For $i=1, \cdots, t$ we construct the algebra $\mathfrak{A}_{i}$ over the powerseries field $\mathfrak{F}\left\{X_{1}, \cdots, X_{i}\right\}$ by setting $\gamma_{k}=X_{k}$ for $k=1, \cdots, i$. Let $\mathfrak{A}_{0}=\widetilde{F}$.

By induction on $i$ we now show that $\mathfrak{A}_{i}$ is a division algebra for $i=1, \cdots, t$. Assume that $\mathfrak{A}_{i-1}$ is a division algebra and suppose that $x=a+b u_{i}$ and $y=c+d u_{i}$ are nonzero elements of $\mathfrak{A}_{i}$ such that $x y=0$. Then

$$
a c+X_{i} d^{*} b=0 \text { and } d a+b c^{*}=0 .
$$

As before, $a, b, c, d$ must be nonzero, and we can write

$$
\begin{aligned}
& a=a_{m} X_{i}^{m}+a_{m+1} X_{i}^{m+1}+\cdots, \\
& b=b_{n} X_{i}^{n}+b_{n+1} X_{i}^{n+1}+\cdots, \\
& c=c_{p} X_{i}^{p}+c_{p+1} X_{i}^{p+1}+\cdots, \\
& d=d_{q} X_{i}^{q}+d_{q+1} X_{i}^{q+1}+\cdots,
\end{aligned}
$$

where all the $a_{k}, b_{k}, c_{k}, d_{k}$ are in $\mathfrak{U}_{i-1}$ and $a_{m}, b_{n}, c_{p}, d_{q}$ are nonzero. By (12), $m+p=1+q+n$ and $m+q=n+p$. Adding these two equations, we obtain $2 m+p+q=2 n+p+q+1$. But 1 is not an 
even integer, so that $\mathfrak{A}_{i}$ must be a division algebra.

\section{REFERENCES}

1. A. A. Albert and N. Jacobson, On reduced exceptional simple Jordan algebras, Ann. of Math. 66 (1957), 400-417.

2. N. Jacobson, Composition algebras and their automorphisms, Rend. Circ. Mat. Palermo. (II) 7 (1958), 1-26.

3. O. T. O'Meara, Introduction to Quadratic Forms, Springer, Berlin, 1963.

4. R. D. Schafer, On the algebras formed by the Cayley-Dickson process, Amer. J. Math. 76 (1954), 435-446.

Received January 23, 1966. Research partially supported by National Science Foundation grant GP-3990.

University of CALifornia, Berkeley 


\title{
PACIFIC JOURNAL OF MATHEMATICS
}

\author{
EDITORS
}

H. Samelson

Stanford University

Stanford, California

J. P. JANS

University of Washington

Seattle, Washington 98105

\section{J. DugundJI}

University of Southern California Los Angeles, California 90007

RICHARD ARENS

University of California

Los Angeles, California 90024

\section{ASSOCIATE EDITORS}

E. F. BECKENBACH

B. H. NeUmanN

F. WOLF

K. YosIDA

\section{SUPPORTING INSTITUTIONS}

UNIVERSITY OF BRITISH COLUMBIA

CALIFORNIA INSTITUTE OF TECHNOLOGY

UNIVERSITY OF CALIFORNIA

MONTANA STATE UNIVERSITY

UNIVERSITY OF NEVADA

NEW MEXICO STATE UNIVERSITY

OREGON STATE UNIVERSITY

UNIVERSITY OF OREGON

OSAKA UNIVERSITY

UNIVERSITY OF SOUTHERN CALIFORNIA

\author{
STANFORD UNIVERSITY \\ UNIVERSITY OF TOKYO \\ UNIVERSITY OF UTAH \\ WASHINGTON STATE UNIVERSITY \\ UNIVERSITY OF WASHINGTON \\ * \\ AMERICAN MATHEMATICAL SOCIETY \\ CHEVRON RESEARCH CORPORATION \\ TRW SYSTEMS \\ NAVAL ORDNANCE TEST STATION
}

Mathematical papers intended for publication in the Pacific Journal of Mathematics should be typewritten (double spaced). The first paragraph or two must be capable of being used separately as a synopsis of the entire paper. It should not contain references to the bibliography. Manuscripts may be sent to any one of the four editors. All other communications to the editors should be addressed to the managing editor, Richard Arens at the University of California, Los Angeles, California 90024.

50 reprints per author of each article are furnished free of charge; additional copies may be obtained at cost in multiples of 50 .

The Pacific Journal of Mathematics is published monthly. Effective with Volume 16 the price per volume (3 numbers) is $\$ 8.00$; single issues, $\$ 3.00$. Special price for current issues to individual faculty members of supporting institutions and to individual members of the American Mathematical Society: $\$ 4.00$ per volume; single issues $\$ 1.50$. Back numbers are available.

Subscriptions, orders for back numbers, and changes of address should be sent to Pacific Journal of Mathematics, 103 Highland Boulevard, Berkeley 8, California.

Printed at Kokusai Bunken Insatsusha (International Academic Printing Co., Ltd.), No. 6, 2-chome, Fujimi-cho, Chiyoda-ku, Tokyo, Japan.

PUBLISHED BY PACIFIC JOURNAL OF MATHEMATICS, A NON-PROFIT CORPORATION

The Supporting Institutions listed above contribute to the cost of publication of this Journal, but they are not owners or publishers and have no responsibility for its content or policies. 


\section{Pacific Journal of Mathematics}

\section{Vol. 20, No. $3 \quad$ November, 1967}

Dallas O. Banks, Lower bounds for the eigenvalues of a vibrating string whose density satisfies a Lipschitz condition ................... 393

Ralph Joseph Bean, Decompositions of $E^{3}$ which yield $E^{3} \ldots \ldots \ldots \ldots 411$

Robert Bruce Brown, On generalized Cayley-Dickson algebras ......... 415

Richard Dowell Byrd, Complete distributivity in lattice-ordered groups . . . 423

Roger Countryman, On the characterization of compact Hausdorff $X$ for

which $C(X)$ is algebraically closed ...................... 433

Cecil Craig, Jr. and A. J. Macintyre, Inequalities for functions regular and bounded in a circle ...................................... 449

Takesi Isiwata, Mappings and spaces ..................... 455

David Lewis Outcalt, Power-associative algebras in which every subalgebra is an ideal ..................................... 481

Sidney Charles Port, Equilibrium systems of stable processes .......... 487

Jack Segal, Quasi dimension type. I. Types in the real line ............ 501

Robert William Stringall, Endomorphism rings of primary abelian

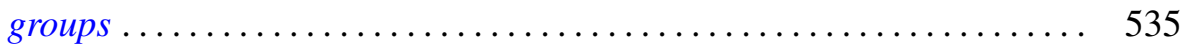

William John Sweeney, "The $\delta$-Poincaré estimate” ................. 559

L. Tzafriri, Operators commuting with Boolean algebras of projections of finite multiplicity ............................... 571 